\title{
Review Article \\ The Unravelled Link between Chronic Kidney Disease and Hepatitis C Infection
}

\author{
Fabrizio Fabrizi, ${ }^{1}$ Piergiorgio Messa, ${ }^{1}$ and Paul Martin ${ }^{2}$ \\ ${ }^{1}$ Division of Nephrology, Maggiore Hospital and IRCCS Foundation, 20122 Milan, Italy \\ ${ }^{2}$ Division of Hepatology, School of Medicine, Miami, FL, USA \\ Correspondence should be addressed to Fabrizio Fabrizi; fabrizi@policlinico.mi.it
}

Received 19 March 2014; Accepted 4 June 2014; Published 3 July 2014

Academic Editor: Alessandro Granito

Copyright (c) 2014 Fabrizio Fabrizi et al. This is an open access article distributed under the Creative Commons Attribution License, which permits unrestricted use, distribution, and reproduction in any medium, provided the original work is properly cited.

\begin{abstract}
The 2011 report of the World Health Organization General Assembly on noncommunicable diseases identified chronic kidney disease as a worldwide health issue posing a heavy economic burden. Hepatitis $\mathrm{C}$ virus infection, which is responsible for over 1 million deaths resulting from cirrhosis and liver cancer, is linked to chronic kidney disease in several ways; some forms of renal disease are precipitated by hepatitis $\mathrm{C}$ and patients with end-stage chronic renal disease are at increased risk for acquiring HCV. The aim of this review is to update the evidence on the relationship between hepatitis $\mathrm{C}$ infection and chronic kidney disease. Information has been accumulated in the last decade indicating that HCV plays an adverse effect on the incidence and progression of chronic kidney disease; a novel meta-analysis of observational studies (seven longitudinal studies; 890,560 unique individuals) found a relationship between hepatitis $C$ seropositivity and incidence of reduced estimated glomerular filtration rate (adjusted relative risk, $1.70 ; 95 \%$ CI, $1.20 ; 2.39 ; P=0.002)$ in the adult general population. In addition to conventional risk factors, hepatitis $\mathrm{C}$ may be an additional factor for the development of chronic kidney disease, and an atheromasic activity of hepatitis $\mathrm{C}$ virus has been mentioned. The link between hepatitis $\mathrm{C}$ and atherosclerosis could also explain the excess risk of cardiovascular mortality that has been observed among hepatitis $\mathrm{C}$ virus seropositive patients undergoing maintenance dialysis. A number of biologically plausible mechanisms related to hepatitis $\mathrm{C}$ virus have been hypothesized to contribute to atherosclerosis. Implementation of effective treatment intervention towards hepatitis $\mathrm{C}$ is required to decrease the healthcare burden of hepatitis $\mathrm{C}$ and to prevent the progression of chronic renal disease.
\end{abstract}

\section{Introduction}

Noncommunicable diseases (NCDs) have replaced communicable diseases as the most common causes of morbidity and premature mortality worldwide $[1,2]$. By 2015, cardiovascular disease and diabetes are expected to reduce global gross domestic product by $5 \%$; this gives emphasis on the enormous global economic impact of NCDs. Four NCDs (cardiovascular disease, cancer, diabetes, and chronic respiratory disease) have been prioritized in the Global NCD Action Plan endorsed by the World Health Assembly in 2008 because they share behavioural risk factors amenable to public health action and, together, represent a major portion of the global NCD burden [3]. Although chronic kidney disease (CKD) is absent from the World Health Organization (WHO) priority NCDs (heart disease, diabetes, chronic respiratory disease, and cancer), compelling information exists that chronic kidney disease is a key determinant of the poor health outcomes of major noncommunicable diseases (particularly diabetes and cardiovascular disease) and the WHO has recently recommended the prevention of CKD within national NCD programs at the primary-care level [4].

The prevalence of CKD, defined by a reduction in glomerular filtration rate and increased urinary albumin excretion, exceeds $10 \%$ of the adult general population [5], and information in the last decade has shown a good association between chronic renal disease and hepatitis $\mathrm{C}$ virus (HCV) infection [6], which has an estimated prevalence of $3 \%$ in the world population (more than 170 million people worldwide chronically infected with HCV) [7]. The association between CKD and HCV occurs by several ways; in addition to conventional risk factors for chronic kidney disease such 
TABLE 1: CKD stages according to eGFR and ACR.

\begin{tabular}{lcc}
\hline CKD stages & eGFR $\left(\mathrm{mL} / \mathrm{min} / 1.73 \mathrm{~m}^{2}\right)$ & ACR $(\mathrm{mg} / \mathrm{g})$ \\
\hline 1 & $\geq 90$ & $\geq 30$ \\
2 & $60-89$ & $\geq 30$ \\
3 & $30-59$ & - \\
4 & $15-29$ & - \\
5 & $<15$ & - \\
\hline
\end{tabular}

ACR: albumin creatinine ratio; CKD: chronic kidney disease; eGFR: estimated glomerular filtration rate.

as aging, metabolic syndrome, arterial hypertension, and diabetes mellitus, HCV infection may be an additional risk factor.

The aim of this review is to update the evidence on the relationship between HCV infection and prevalence (or severity) of chronic kidney disease; the most recent insights on HCV infection both as a cause and complication of chronic kidney disease have been reviewed.

\section{Chronic Kidney Disease: Definition and Clinical Implications}

The definition of CKD has been made first in 2002 when the medical community agreed on a uniform definition (and staging) of chronic renal disease, based on measures of kidney function and independent of the cause of impaired kidney function [8]. The rising incidence and prevalence of chronic renal failure and its associated high costs and poor outcomes prompted nephrologists to issue international guidelines to define chronic kidney disease. As listed in Table 1, chronic kidney disease has been defined by reduced glomerular filtration rate $\left(<60 \mathrm{~mL} / \mathrm{min} / 1.73 \mathrm{~m}^{2}\right)$ for three months or more and/or proteinuria [8].

Chronic renal disease has been recognized as a worldwide health problem that affects $10-15 \%$ of the adult general population worldwide, and the overall prevalence of CKD at least equals that of diabetes. According to the 1999-2004 National Health and Nutrition Examination Survey (NHANES) III data, the prevalence of CKD was $16.8 \%$ in the US population aged $\geq 20$ years, compared with $14.5 \%$ from the $1988-1994$ NHANES, suggesting that CKD is a growing health problem in the United States [13]. NHANES is a nationally representative sample of noninstitutionalized adults living in the USA and aged 20 years or older $(n=13,233)$. As with many other chronic diseases, the prevalence of CKD increases with age, exceeding 20\% in individuals older than 60 years and $35 \%$ in those older than 70 years.

The clinical and public health importance of CKD became soon apparent when large surveys showed that even early stage kidney disease is associated with adverse outcomes including increased risk of mortality and cardiovascular morbidity. Go et al. [14] in a large, community-based population ( $n=1,120,295$ adults) observed that the graded decrease of estimated glomerular filtration rate (GFR) was independently associated with the adjusted hazard ratio (aHR) for death, cardiovascular events, and hospitalization. The aHR for death was $1.2(95 \%$ CI, 1.1; 1.2) with an estimated GFR of 45 to $59 \mathrm{~mL} / \mathrm{min}, 1.8(95 \% \mathrm{CI}, 1.7 ; 1.9)$ with an eGFR of 30 to $44 \mathrm{~mL} / \mathrm{min}, 3.2$ (95\% CI, 3.1-3.4) with an estimated GFR of 15 to $29 \mathrm{~mL} / \mathrm{min}$, and $5.9(95 \% \mathrm{CI}, 5.4 ; 6.5)$ with an estimated GFR less than $15 \mathrm{~mL} / \mathrm{min}$. CKD is also associated with decreased quality of life, cognitive dysfunction, and increased health-care expenditures $[1,2]$.

\section{Chronic Kidney Disease and Its Global Perspective}

One potential outcome of chronic kidney disease is endstage renal disease (ESRD) which requires renal replacement therapy (i.e., maintenance dialysis and renal transplantation). Renal replacement therapy is very costly; thus, ESRD is an important cost driver for health-care systems of developed countries and over 2 million people now need renal replacement therapy to sustain life. The majority of patients undergoing renal replacement treatment are in five countries (Brazil, Italy, Japan, Germany, and the USA) that constitute $12 \%$ of the world population. $20 \%$ undergo renal replacement therapy in about 100 developing countries that make up over $50 \%$ of the world population. These data highlight the relationship between gross domestic product and availability of renal replacement therapy; also, the high burden of ESRD and associated costs is emphasized.

Over the past 15 years, evidence from clinical trials and meta-analyses has shown that the presence of CKD dramatically increases the risk of adverse outcomes among people with other NCDs. Chronic renal disease strongly predisposes to hypertension [15] and cardiovascular disease [16]; diabetes [17], hypertension [18], and cardiovascular disease [19] are all major causes of CKD; major risk factors for diabetes [1], hypertension [1], and cardiovascular disease (such as obesity, and smoking) [20] also cause or exacerbate chronic renal disease; finally, treatments for slowing progression of CKD also reduce complications from diabetes, hypertension, and cardiovascular disease [21].

All acute and chronic diseases have a worse prognosis in the presence of kidney disease, and this is of particular relevance for cardiovascular diseases such as stroke, heart failure, and acute myocardial infarction [5]. Chronic renal disease also worsens the prognosis of patients with diabetes and pulmonary and metabolic disease. Presence of kidney disease affects patient management; for example, patients with kidney disease can experience restrictions in the use of imaging procedures requiring the application of radiocontrast agents or gadolinium and this can lead to underdiagnosis in patients with CKD [5]. Uncertainty in drug dosing including drug toxicity and underdosing is another potential source of dismal prognosis in CKD population.

Given that CKD is usually asymptomatic until later stages of the disease (stage 4 or more), a lack of awareness of earlier stages of CKD exists and risk factors for CKD are frequently underrecognized. The lack of awareness may extend to clinical physicians. Prior studies have demonstrated that early identification and management of CKD reduce the risk of progression to kidney failure and cardiovascular disease by up to $50 \%$ [22]. 


\section{HCV-Associated Kidney Disease}

In addition to conventional risk factors, other causes of chronic renal disease including drugs or alternative medications have been cited. The role of HCV in the course, morbidity, and mortality of renal disease is often not appropriately considered in the care of patients with CKD; however, HCV infection has been proposed as a possible cause of CKD. HCV has been identified in 1989 and was recognized soon as the main cause of non-A and non-B hepatitis [7]. In Western Europe, $\mathrm{HCV}$ prevalence ranges from 0.4 to $3 \%$; prevalence is higher in Eastern Europe and the Middle East, up to 13\% in Egypt. More than 170 million people worldwide are chronically infected with the hepatitis $\mathrm{C}$ virus (HCV) which is currently one of the major causes of chronic liver disease worldwide and remains a major global health burden. The long-term hepatic impact of HCV infection includes chronic hepatitis and cirrhosis, with or without hepatocellular carcinoma (HCC). Several extrahepatic manifestations have been associated with HCV infection, including haematological, dermatologic, autoimmune, and kidney diseases. HCV infection is frequently asymptomatic; thus, most chronically infected individuals have no knowledge of their infection [7].

There is increasing evidence of an association between $\mathrm{HCV}$ infection and glomerular disease in both native kidneys [23-25] and after kidney [26] or liver [27, 28] transplant. The most common type of HCV-related glomerulonephritis is type 1 membranoproliferative glomerulonephritis usually in the context of type II cryoglobulinemia [29]. HCVinfected patients show less common glomerular diseases including membranoproliferative glomerulonephritis without cryoglobulinemia, membranous nephropathy, focal segmental sclerosis, mesangial glomerulonephritis, and fibrillary and immunotactoid glomerulopathies. Anecdotal information has been reported on HCV-associated IgA nephropathy and cryoglobulinemic thrombotic microangiopathy [29]. In addition to glomerular disease, tubulointerstitial injury has been also observed in association with HCV infection [30]. Various surveys have evaluated the frequency of antiHCV seropositive status in patients with biopsy-proven glomerulonephritis [31, 32] but the most important report on the epidemiology of HCV-associated glomerular disease has been conducted by El-Serag et al. [33]. They carried out a hospital-based case control study that enrolled all cases of HCV-infected patients hospitalized during the 1990s $(n=34,204)$ and randomly chosen control male individuals without HCV $(n=136,816)$ matched with cases on the year of admission. As expected, cryoglobulinemia was more common in cases than controls $(0.57 \%$ versus $0.05 \%, P<$ $0.0001)$, and a greater prevalence of membranoproliferative glomerulonephritis $(0.36 \%$ versus $0.05 \%)$ but not membranous nephropathy $(0.33 \%$ versus $0.19 \%)$ was observed in $\mathrm{HCV}$-infected individuals.

A recent meta-analysis of clinical studies (four clinical studies with 81,286 unique individuals) demonstrated that HCV seropositive status was an independent and significant risk factor for proteinuria in the general population, with a summary estimate for adjusted relative risk of 1.47 (95\% CI, 1.12; 1.94; $P=0.006)$. Significant heterogeneity occurred
$(R i=0.82 ; P$-value by $Q$ test, <0.001) [34]. Proteinuria clusters with the metabolic syndrome and surveys have reported a relationship between proteinuria and individual components of the metabolic syndrome (insulin resistance, hyperglycemia, dyslipidemia, abdominal obesity, and arterial hypertension). Because patients with $\mathrm{HCV}$ are known to have higher prevalence of components of the metabolic syndrome, it has been hypothesized that individuals with HCV may have high frequency of proteinuria. The persistence of the relationship between $\mathrm{HCV}$ and proteinuria after correction for several confounding parameters including metabolic syndrome elements is in keeping with the occurrence of $\mathrm{HCV}$-associated cryoglobulinemic or intrinsic renal disease.

The main clinical manifestations of $\mathrm{HCV}$-associated glomerular disease are the presence of proteinuria and microscopic hematuria with or without impaired kidney function. It has been recommended by the KDIGO HCV guidelines that patients infected with $\mathrm{HCV}$ be tested at least annually for proteinuria, hematuria, and eGFR [29]. HCVinfected patients with clinical evidence of glomerulonephritis should undergo kidney biopsy and the outcome of HCVassociated glomerulonephritis is improved by early diagnosis and therapy. The routine laboratory diagnosis of HCV-related glomerulonephritis is based on the presence of conventional markers of HCV infection such as anti-HCV antibody and $\mathrm{HCV}$ viraemia (HCV RNA). The disappearance of proteinuria after interferon therapy with clearance of HCV RNA from serum strongly suggests the pathogenic role of HCV in this type of glomerular disease [29].

Studies are under way to assess whether HCV-associated glomerular disease could be related to occult HCV infection (i.e., detectable viral RNA in peripheral blood mononuclear cells or in serum after ultracentrifugation); occult $\mathrm{HCV}$ occurs only in anti-HCV negative patients with no detectable $\mathrm{HCV}$ RNA in serum. Occult HCV infection has been observed in various patient groups such as hemodialysis patients, patients with cryptogenic hepatitis and liver cirrhosis, hepatocellular carcinoma, lymphoproliferative disorders, and healthy individuals without evidence of liver disease. The role played by occult HCV infection in the development of $\mathrm{HCV}$-associated glomerular disease is in keeping with some pieces of evidence; for example, interferon based therapy has given benefit in some cases of HCV negative cryoglobulinemic glomerulonephritis [35]. HCV antigens or particles have been found in the kidney tissue of patients with HCV negative glomerulonephritis [36]. If confirmed, occult HCV infection could be the underlying cause of glomerular disease in some patients with negative serologic viral markers, and detection of occult HCV might be the rationale for antiviral therapy in anti-HCV negative glomerular nephropathy.

\section{Mechanisms of HCV-Associated Renal Injury}

The mechanisms of HCV-related renal disease are uncertain and research has suggested various causes including a direct cytopathic activity of HCV on renal parenchima [37]. Renal parenchima expresses CD8 and SR-B1 receptors that 
allow binding of HCV to the cell surface and endocytosis. Hepatitis $C$ virus-related proteins have been identified in mesangial cells, tubular epithelial cells, and endothelial cells of glomerular and tubular capillaries [38]. An association between greater proteinuria and mesangial deposition of HCV RNA and related proteins has been suggested; this possibly mirrors a direct mesangial injury by HCV [39].

Much more is known about kidney injury due to systemic immune response to $\mathrm{HCV}$ infection that is mediated by cryoglobulins (a group of globulins with the common property of precipitating from cooled serum). Persistent HCV infection gives overstimulation of B-lymphocytes and production of mixed cryoglobulins which are mainly composed of a polyclonal immunoglobulin (Ig), either IgG or IgM, bound to another Ig that works as an antirheumatoid factor (RF) [4042]. HCV-associated glomerular injury can result from deposition of circulating immune complexes containing $\mathrm{HCV}$ proteins, anti-HCV antibody, and rheumatoid factor IgM. Cryoglobulins are frequently deposited in the mesangium and their nephrotoxicity is related to affinity of the IgM- $k$-RF for cellular fibronectin in the mesangial matrix. Deposition of cryoglobulins in the glomerular capillaries appears as eosinophilic material which is stained with antisera to $\operatorname{IgM}$, C3, and fibrin by immunofluorescence. Cryoglobulins may also induce endothelitis via anti-endothelial antibody activity and complement activation; an overexpression of VCAM1 has been found with platelet aggregation. Viral HCV antigens have been found by immunochemistry and by in situ hybridization. Some authors have used laser microdissection to detect HCV RNA genomic sequences of HCV core protein in glomeruli and tubules of patients with $\mathrm{HCV}$-associated glomerular disease [39].

Recently, an elevation of toll-like receptor 3 (TLR3) messenger RNA expression has been seen in glomeruli, particularly mesangial cells, of patients with HCV-induced $\mathrm{GN}$, this being associated with an increased activity of some proinflammatory cytokines. A greater TLR3 expression was observed in peripheral mononuclear cells of patients with $\mathrm{HCV}$ positive GN $(n=46)$ than $\mathrm{HCV}$ negative patients $(n=32)$ or healthy individuals $(n=20), P<0.0001$ [43]. TLRs are primary proteins expressed on immune and nonimmune cells as key components of the innate immune system. TRLs recognize molecular patterns associated with microbial agents and give an immune response [44].

Nonimmunologically mediated mechanisms of kidney disease have been also hypothesized. Anti-HCV positive patients, with or without cirrhosis, show raised levels of fasting serum insulin and insulin resistance and greater prevalence of diabetes mellitus. HCV core protein directly reduces expression of insulin receptor substrate proteins (IRS) 1 and 2 which are involved in the metabolic activities of insulin and stimulate activation of IRS- 1 and high expression of TNF- $\alpha$ at least in hepatic cells $[45,46]$. Insulin resistance and hyperinsulinemia give excess intrarenal synthesis of IGF1 and $\operatorname{TGF} \beta$, thus promoting proliferation of renal cells and upregulating the expression of angiotensin II type 1 receptors in mesangial cells. The deleterious activities of angiotensin II at kidney level are enhanced. An excess local production of endothelin-1, reduced endothelial synthesis of nitric oxide, and increased oxidative stress have been implicated in the pathogenesis and progression of kidney damage.

\section{HCV and Chronic Kidney Disease: Evidence from the General Population}

Renal involvement of hepatitis $C$ virus infection was first reported two decades ago; however, knowledge of the association between HCV and low eGFR in the adult general population is limited and controversial. The prevalence of $\mathrm{HCV}$ among patients with CKD stage 5 (including patients on maintenance dialysis) is much higher than for the general population, and it is unknown whether this reflects an increased risk of viral exposure, a greater incidence and progression of kidney disease in individuals with $\mathrm{HCV}$, or both.

The association between HCV infection with the prevalence and severity of chronic kidney disease in the adult general population has been addressed in several surveys derived from large databases (Table 2) [47-49]. Various cohort studies from developed countries have been published, some studies reported a positive association of HCV and CKD in the presence of cirrhosis in a population of veterans [12] or among national health insurance program users [50], but no such associations were identified in another population of veterans [9], an urban hospital population [10], and a private health insurance population [11]. Other cross-sectional surveys from the United States [51] and southern Taiwan [52] have yielded conflicting findings. We have performed a systematic review with a meta-analysis of observational studies (nine clinical studies; 817,917 unique individuals) to determine whether $\mathrm{HCV}$ is associated with increased likelihood of kidney disease in the general population [34]. We retrieved only studies performing multivariate analysis to provide the independent effect of anti-HCV serologic status on the incidence or prevalence of an estimated GFR $\left(<60 \mathrm{~mL} / \mathrm{min}\right.$ per $\left.1.73 \mathrm{~m}^{2}\right)$ in the general population. Pooling of study results demonstrated the absence of a relationship between positive anti-HCV serologic status and reduced GFR $\left(<60 \mathrm{~mL} / \mathrm{min} / 1.73 \mathrm{~m}^{2}\right)$ in the general population (adjusted relative risk (aRR), 1.12; 95\% CI, 0.91; 1.38; $P=0.28$ ) according to the randomeffects model [34]. We have now updated our systematic review of the published medical literature, and we retrieved longitudinal studies ( $n=7 ; 890,560$ unique individuals). A relationship between anti-HCV seropositivity and incidence of reduced eGFR (adjusted relative risk, 1.70; 95\% CI, 1.20; 2.39; $P=0.002)$ in the adult general population was noted; substantial heterogeneity $(R i=0.98 ; P$-value by $Q$-test, $<0.0001$ ) was detected without publication bias (Figure 1). Thus, these findings support consistently an adverse effect of HCV on the development of chronic kidney disease in the general population. The most important shortcoming of these meta-analyses is that all the studies included had an observational design. Although much has been learned on the outcome of $\mathrm{HCV}$ in patients with chronic kidney disease or intact kidney function, the available data are of limited nature due to the lack of controlled studies that provide baseline data and sequential follow-up, in patients 
TABLE 2: Impact of HCV on eGFR in the adult general population.

\begin{tabular}{|c|c|c|c|c|}
\hline Author & Patient, $n$ & aRR & Country & Reference year \\
\hline Tsui et al. [9] & 474,369 & $2.80(2.43 ; 3.23)$ & Taiwan & 2007 \\
\hline Moe et al. [10] & 7,038 & $1.02(0.90 ; 1.15)$ & US & 2008 \\
\hline Asrani et al. [11] & 88,822 & $0.92(0.79 ; 1.08)$ & US & 2010 \\
\hline Butt et al. [12] & 43,139 & $1.3(1.23 ; 1.37)$ & US & 2011 \\
\hline Hofmann J. et al. & 223,536 & $4.6(3.7 ; 5.8)$ & Sweden & 2011 \\
\hline Su F. et al. & 37,746 & $1.53(1.17 ; 2.01)$ & Taiwan & 2012 \\
\hline Chen Y. et al. & 15,910 & $1.75(1.25 ; 2.43)$ & Taiwan & 2013 \\
\hline
\end{tabular}

eGFR: estimated glomerular filtration rate.

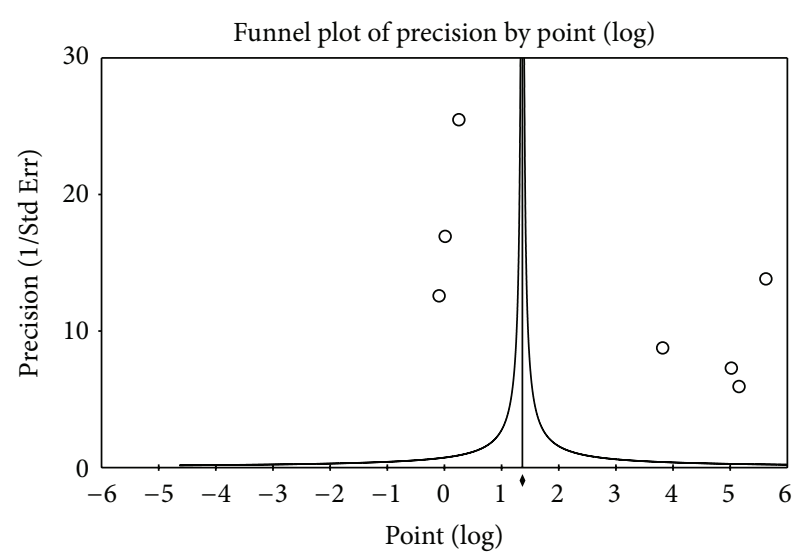

FIGURE 1: Funnel plot of precision by point (log).

with initially intact kidney function and HCV. These metaanalyses are potentially biased by additional issues. The crosssectional design of many studies did not allow drawing conclusions on causality; also, the studies included in these metaanalyses might give incomplete information on additional unmeasured confounders that could introduce bias into the analysis. A magnitude of missing data or insensitive codes for comorbid diagnoses are inherent to clinical databases opposed to research databases. Additional limitations are that most surveys were from the United States and Taiwan and various populations (including veterans or inner city residents) have been evaluated. Finally, we cannot rule out an underestimation of negative results related to publication bias (positive relationships published more than negative ones).

\section{HCV and Development of Chronic Kidney Disease: Additional Evidence}

The detrimental role of $\mathrm{HCV}$ on the progression of chronic renal disease has been observed from sources other than the adult general population. Cohort studies performed in diabetic patients, individuals with HIV and HCV coinfection, and cirrhotics who completed IFN therapy demonstrated a relationship between positive anti-HCV serologic status and accelerated progression of chronic kidney disease. Soma et al. [53] evaluated 123 patients with biopsy-proven type II diabetic-related glomerulosclerosis (19.5\%) over a follow-up period of $27.6 \pm 13.3$ months; anti-HCV positive serologic status was an independent and significant risk factor of progression of chronic kidney disease by multivariable analysis $(-0.1828,95 \% \mathrm{CI},-0.2787 ;-0.0869 ; P<0.0001)$.

Mocroft et al. [54] found that the odds of progressive CKD were increased in participants with HCV (adjusted OR, 1.72; 95\% CI, 1.07; 2.76) among 3,441 HIV positive, antiretroviral-treated clinical trial participants. Progressive CKD was defined as the composite of end-stage renal disease, renal death, or significant glomerular filtration rate (eGFR) decline $\left(25 \%\right.$ decline to eGFR $<60 \mathrm{~mL} / \mathrm{min} / 1.73 \mathrm{~m}^{2}$ or $25 \%$ decline with a baseline $<60 \mathrm{~mL} / \mathrm{min} / 1.73 \mathrm{~m}^{2}$ ). In addition to conventional risk factors for chronic kidney disease such as aging, obesity, arterial hypertension, and diabetes mellitus, HCV infection may be an additional factor. This view is promoted by the notion that conventional risk factors only partially explain the excess risk of chronic kidney disease in the general population.

In the survey of Arase et al. [55], 650 patients with HCVrelated cirrhosis were followed up for a mean period of 6.5 years after completing IFN therapy. Nonclearance of HCV was linked to the development of chronic kidney disease (eGFR of $<60 \mathrm{~mL} / \mathrm{min}$ per $1.73 \mathrm{~m}^{2}$ ), adjusted HR, $2.67(95 \%$ CI, $1.34 ; 5.32, P=0.005)$ [13].

\section{HCV and Development of Chronic Kidney Disease: Potential Mechanisms}

Various mechanisms have been cited to explain the adverse impact of anti-HCV seropositive status on the development of chronic kidney disease in the adult general population. An accelerated atheromasic disease at kidney level instigated by HCV has been mentioned, among others. The atherogenic activity of HCV has been also suggested to explain the adverse impact of HCV on the survival of patients undergoing maintenance dialysis. We have recently performed a metaanalysis of observational studies (fourteen observational studies involving 145,608 unique patients on long-term dialysis); pooling of study results demonstrated that anti-HCV antibody was an independent and significant risk factor for death in patients on regular dialysis. The summary estimate for adjusted relative risk (all-cause mortality) was 1.35 with a $95 \%$ confidence interval (CI) from 1.25 to 1.47 . Stratified analysis showed that the adjusted relative risk ( $\mathrm{aRR}$ ) for liver disease-related and cardiovascular mortality was 3.82 (95\% 
CI, 1.92; 7.61) and 1.26 (95\% CI, 1.1; 1.45), respectively [56]. Only three reports gave detailed information on the relationship between anti-HCV serologic status and cardiovascularrelated mortality, however, the size of the subgroup of studies was large $(n=37,787)$ with no significant heterogeneity. Thus, the excess risk of death in anti-HCV positive patients is related either to chronic liver disease (and its long-term complications such as cirrhosis and hepatocellular carcinoma) and cardiovascular mortality. Because patients on long-term dialysis have an exceptionally high short-term mortality, it is less likely that the HCV-associated death risk is only due to such long-term complications as liver disease. The excess risk of cardiovascular disease among anti-HCV positive patients undergoing maintenance dialysis has been attributed to an atherogenic role of HCV through the aggravation of individual components of the metabolic syndrome (hyperglycemia, insulin resistance, abdominal obesity, arterial hypertension, and dyslipidemia).

That HCV could prompt cardiovascular alterations in patients with chronic kidney disease (via direct and indirect mechanisms) has been suggested by the epidemiological evidence reported above and other additional data. Yelken et al. [57] measured coronary flow reserve (CFR) by transthoracic Doppler echocardiography in nondiabetic anti-HCV positive $(n=26)$ and negative $(n=26)$ patients undergoing regular dialysis (haemodialysis or peritoneal dialysis) after kidney transplant failure. Clinical and demographic characteristics were similar between the two groups. CFR was significantly lower in HCV-infected than uninfected individuals $(1.5 \pm 1.1$ versus $1.63 \pm 0.26, P=0.03$ ).

Oyake and colleagues [58] evaluated 94 dialysis patients (17 being anti-HCV positive/HCV RNA positive) by prospective measurements of aortic stiffness using carotid-femoral pulse wave velocity (PWV). They found by multiple logistic regression analysis that mean blood pressure and $\mathrm{HCV}$ viremia (OR, 9.7; 95\% CI, 1.18; 81.2, $P<0.05)$ were significantly and independently associated with high aortic PWV ( $>$ or $=10.0 \mathrm{~m} / \mathrm{sec}$, mean). During the follow-up period, 13 patients suffered from cardiovascular events. Kaplan-Meier survival curves for cerebrovascular and cardiovascular eventfree rates indicated a highly significant difference between HCV-RNA positive or negative patients on dialysis (log-rank test, $P<0.05$ ). The authors mentioned an atherogenic role of HCV through aggravation of metabolic syndrome and dyslipidemia.

\section{HCV and Cardiovascular Diseases in the General Population: Novel Data}

$\mathrm{HCV}$ infection is currently considered by some investigators as a systemic disease, for which clinicians must evaluate the anti-HCV positive patient either for liver disease and cardiovascular or cerebrovascular risk [59]. The strongest evidence of a relationship between chronic HCV infection and cardiovascular or cerebrovascular events in the adult general population has been generated by longitudinal studies evaluating the natural history of patients with chronic HCV. At least three prospective studies have found a significant increase in cardiovascular mortality [60], frequency of stroke [61], and lethal cerebrovascular events [62], respectively, in anti-HCV positive compared to negative individuals with intact kidney function. In addition, at least four retrospective surveys have reported an excess in acute coronary artery syndrome [63], incidental coronary artery disease [64], stroke [65], and cardiovascular mortality [66] in anti-HCV positive compared to negative patients. Only one retrospective cohort study concluded that HCV was not an independent risk factor for acute myocardial infarction among adult followed in general practices in the United Kingdom [67].

The evidence coming from case control studies is more equivocal. Many authors found a relationship between HCV and carotid atherosclerosis [68-71], others a link between HCV and coronary atherosclerosis [72, 73] or myocardial dysfunction $[74,75]$. However, some conflicting reports have been given [76-79]. Discrepancies between the observed results could likely be due to the characteristics of the studied populations and differences in the assessment of $\mathrm{HCV}$ infection (or its severity) or in the evaluation of carotid or coronary atherosclerosis. The potential confounding by unmeasured or inadequately controlled variables cannot be excluded.

Recent data from the medical literature suggest that HCV could prompt cardiovascular and peripheral vascular disease by multiple ways. Some metabolic alterations (i.e., obesity, insulin resistance, diabetes, steatosis, and dyslipidemia) have been reported more frequently in HCV infected compared with uninfected patients, due to the interferences played by HCV in the glucose and lipid metabolism [80-82]. Insulin resistance has been associated with endothelial dysfunction, abnormalities in nitric oxide production, and platelet dysfunction [83]; type 2 diabetes increases the cardiovascular risk via various mechanisms including increased platelet activity, foam cell formation, or glycation of the extracellular matrix, all of which can increase the risk for atherosclerosis [84]. In addition to these metabolic alterations, alternative mechanisms have been cited. It has been suggested that other infectious agents, via microinflammation, are involved in the pathogenesis of atherosclerotic lesions. As an example, Lin et al. performed a multistage sampling survey in Taiwan $(n=$ 3352 participants, 687 with CKD); they found an independent and significant relationship between HBsAg seropositive status and reduced eGFR (adjusted OR, 1.35, 95\% CI, 1.03; 1.77) and suggested that chronic infections per se may have an adverse impact on the progression of chronic kidney diseases [52]. Boddi et al. [85] detected positive strand HCV RNA in carotid plaques from anti-HCV positive patients but not in plaques of anti-HCV negative individuals and suggested an active local activity of HCV in the vascular tissue. In HCV chronically infected patients, the pathogenesis of liver damage is mostly immune mediated as the immune system is inadequate to eradicate the virus, and atherosclerotic lesions should be the result of the systemic activation of the immune system leading to chronic inflammation via both innate natural killer mediated and adaptive $\mathrm{T}$ helper 1 mediated responses [59]. 


\section{Conclusions}

Chronic renal disease is an important public health problem which significantly increases the likelihood of adverse outcomes and high health-care costs. HCV infection is both a cause and complication of chronic kidney disease, and recent data suggest a detrimental role of HCV in the development of chronic kidney disease in the adult general population. A number of studies reported a high frequency of metabolic comorbidities in anti-HCV positive patients which could at least partially explain the link between HCV seropositive status and a higher prevalence and severity of chronic kidney disease. The future availability of effective and safe therapies could lead to antiviral treatment of HCV infection both to cure liver damage and reduce extrahepatic complications of $\mathrm{HCV}$, including the progression of CKD to late stages.

\section{Conflict of Interests}

The authors declare that there is no conflict of interests regarding the publication of this paper.

\section{References}

[1] W. G. Couser, G. Remuzzi, S. Mendis, and M. Tonelli, "The contribution of chronic kidney disease to the global burden of major noncommunicable diseases," Kidney International, vol. 80, no. 12, pp. 1258-1270, 2011.

[2] Q. Zhang and D. Rothenbacher, "Prevalence of chronic kidney disease in population-based studies: systematic review," $B M C$ Public Health, vol. 8, article 117, 2008.

[3] "2008-2013 action plan for the global strategy for the prevention and control of non-communicable diseases: prevent and control of cardiovascular diseases, cancer, chronic respiratory diseases, and diabetes," World Health Organization.

[4] P. W. Eggers, "Has the incidence of end-stage renal disease in the USA and other countries stabilized?" Current Opinion in Nephrology and Hypertension, vol. 20, no. 3, pp. 241-245, 2011.

[5] K. Eckardt, J. Coresh, O. Devuyst et al., "Evolving importance of kidney disease: from subspecialty to global health burden," The Lancet, vol. 382, no. 9887, pp. 158-169, 2013.

[6] N. Perico, D. Cattaneo, B. Bikbov, and G. Remuzzi, "Hepatitis $\mathrm{C}$ infection and chronic renal diseases," Clinical Journal of the American Society of Nephrology, vol. 4, no. 1, pp. 207-220, 2009.

[7] S. Pol, A. Vallet-Pichard, M. Corouge, and V. O. Mallet, "Hepatitis C: epidemiology, diagnosis, natural history and therapy," Contributions to Nephrology, vol. 176, pp. 1-9, 2012.

[8] National Kidney Foundation, "K/DOQI clinical practice guidelines for chronic kidney disease: evaluation, classification, and stratification," American Journal of Kidney Diseases, vol. 39, supplement 1, pp. S1-S266, 2002.

[9] J. I. Tsui, E. Vittinghoff, M. G. Shlipak et al., "Association of hepatitis $\mathrm{C}$ seropositivity with increased risk for developing end-stage renal disease," Archives of Internal Medicine, vol. 167, no. 12, pp. 1271-1276, 2007.

[10] S. M. Moe, A. J. Pampalone, S. Ofner, M. Rosenman, E. Teal, and S. L. Hui, "Association of hepatitis $\mathrm{C}$ virus infection with prevalence and development of kidney disease," The American Journal of Kidney Diseases, vol. 51, no. 6, pp. 885-892, 2008.

[11] S. K. Asrani, P. Buchanan, B. Pinsky, L. R. Rey, M. Schnitzler, and F. Kanwal, "Lack of association between hepatitis $\mathrm{C}$ infection and chronic kidney disease," Clinical Gastroenterology and Hepatology, vol. 8, no. 1, pp. 79-84, 2010.

[12] A. A. Butt, X. Wang, and L. F. Fried, "HCV infection and the incidence of CKD," American Journal of Kidney Diseases, vol. 57, no. 3, pp. 396-402, 2011.

[13] Centers for Disease Control and Prevention (CDC), "Prevalence of chronic kidney disease and associated risk factorsUnited States, 1999-2004," Morbidity and Mortality Weekly Report (MMWR), vol. 56, pp. 161-165, 2007.

[14] A. S. Go, G. M. Chertow, D. Fan, C. E. McCulloch, and C. $\mathrm{Hsu}$, "Chronic kidney disease and the risks of death, cardiovascular events, and hospitalization," The New England Journal of Medicine, vol. 351, no. 13, pp. 1296-1305, 2004.

[15] S. Klahr, "The kidney in hypertension-villain and victim," The New England Journal of Medicine, vol. 320, no. 11, pp. 731-733, 1989.

[16] C. A. Herzog, R. W. Asinger, A. K. Berger et al., "Cardiovascular disease in chronic kidney disease. A clinical update from Kidney Disease: Improving Global Outcomes (KDIGO)," Kidney International, vol. 80, no. 6, pp. 572-586, 2011.

[17] H. H. Parving, E. Hommel, E. Mathiesen et al., "Prevalence of microalbuminuria, arterial hypertension, retinopathy and neuropathy in patients with insulin dependent diabetes," British Medical Journal, vol. 296, no. 6616, pp. 156-160, 1988.

[18] P. M. Kearney, M. Whelton, K. Reynolds, P. Muntner, P. K. Whelton, and J. He, "Global burden of hypertension: analysis of worldwide data," The Lancet, vol. 365, no. 9455, pp. 217-223, 2005.

[19] W. M. McClellan, R. D. Langston, and R. Presley, "Medicare patients with cardiovascular disease have a high prevalence of chronic kidney disease and a high rate of progression to end-stage renal disease," Journal of the American Society of Nephrology, vol. 15, no. 7, pp. 1912-1919, 2004.

[20] A. Betriu, M. Martinez-Alonso, M. Arcidiacono et al., "Prevalence of subclinical atheromatosis and associated risk factors in chronic kidney disease: the NEFRONA study," Nephrology, Dialysis, Transplantation, vol. 29, no. 7, pp. 1415-1422, 2014.

[21] G. Remuzzi, P. Ruggenenti, and N. Perico, "Chronic renal diseases: renoprotective benefits of renin-angiotensin system inhibition," Annals of Internal Medicine, vol. 136, no. 8, pp. 604$615,2002$.

[22] M. T. James, B. R. Hemmelgarn, and M. Tonelli, "Early recognition and prevention of chronic kidney disease," The Lancet, vol. 375, no. 9722, pp. 1296-1309, 2010.

[23] F. Fabrizi, P. Colucci, C. Ponticelli, and F. Locatelli, "Kidney and liver involvement in cryoglobulinemia," Seminars in Nephrology, vol. 22, no. 4, pp. 309-318, 2002.

[24] F. Fabrizi, E. Plaisier, D. Saadoun, P. Martin, P. Messa, and P. Cacoub, "Hepatitis $C$ virus infection, mixed cryoglobulinemia, and kidney disease," The American Journal of Kidney Diseases, vol. 61, no. 4, pp. 623-637, 2013.

[25] F. Fabrizi, G. B. Fogazzi, D. Cresseri et al., "Antiviral therapy for HCV-associated cryoglobulinemic glomerulonephritis: case report and review of the literature," Kidney and Blood Pressure Research, vol. 35, no. 6, pp. 687-693, 2013.

[26] J. M. Cruzado, M. Carrera, J. Torras, and J. M. Grinyó, “Hepatitis $\mathrm{C}$ virus infection and de novo glomerular lesions in renal allografts," American Journal of Transplantation, vol. 1, no. 2, pp. 171-178, 2001.

[27] M. F. Donato, G. Banfi, D. Cresseri et al., "Antiviral therapy of symptomatic HCV-mixed cryoglobulinemia after liver 
transplant: case report and literature review," The International Journal of Artificial Organs, vol. 36, no. 5, pp. 367-372, 2013.

[28] M. F. Donato, F. Fabrizi, G. B. Fogazzi et al., "Remission of HCVassociated glomerulonephritis with pegylated IFN and ribavirin therapy after liver transplantation: case report and literature review," International Journal of Artificial Organs, vol. 36, no. 1, pp. 63-68, 2013.

[29] KDIGO, "KDIGO clinical practice guidelines for the prevention, diagnosis, evaluation, and treatment of hepatitis $\mathrm{C}$ in chronic kidney disease," Kidney International, vol. 73, no. 109, supplement, pp. S1-S99, 2008.

[30] K. Kasuno, T. Ono, A. Matsumori et al., "Hepatitis C virusassociated tubulointerstitial injury," American Journal of Kidney Diseases, vol. 41, no. 4, pp. 767-775, 2003.

[31] Y. Arase, K. Ikeda, N. Murashima et al., "Glomerulonephritis in autopsy cases with hepatitis C virus infection," Internal Medicine, vol. 37, no. 10, pp. 836-840, 1998.

[32] F. Fabrizi, C. Pozzi, M. Farina et al., "Hepatitis C virus infection and acute or chronic glomerulonephritis: an epidemiological and clinical appraisal," Nephrology Dialysis Transplantation, vol. 13, no. 8, pp. 1991-1997, 1998.

[33] H. B. El-Serag, H. Hampel, C. Yeh, and L. Rabeneck, "Extrahepatic manifestations of hepatitis $\mathrm{C}$ among United States male veterans," Hepatology, vol. 36, no. 6, pp. 1439-1445, 2002.

[34] F. Fabrizi, P. Martin, V. Dixit, and P. Messa, "Hepatitis C virus infection and kidney disease: a meta-analysis," Clinical Journal of the American Society of Nephrology, vol. 7, no. 4, pp. 549-557, 2012.

[35] M. Casato, B. Lagana, L. P. Pucillo, and I. Quinti, "Interferon for hepatitis $C$ virus-negative type II mixed cryoglobulinemia," The New England Journal of Medicine, vol. 338, no. 19, pp. 1386-1387, 1998.

[36] D. Sansonno, G. Lauletta, M. Montrone, G. Grandaliano, F. P. Schena, and F. Dammacco, "Hepatitis C virus RNA and core protein in kidney glomerular and tubular structures isolated with laser capture microdissection," Clinical and Experimental Immunology, vol. 140, no. 3, pp. 498-506, 2005.

[37] R. S. Barsoum, "Hepatitis C virus: from entry to renal injuryfacts and potentials," Nephrology Dialysis Transplantation, vol. 22, no. 7, pp. 1840-1848, 2007.

[38] E. Rodríguez-Iñigo, M. Casqueiro, J. Bartolomé et al., "Hepatitis $\mathrm{C}$ virus RNA in kidney biopsies from infected patients with renal diseases," Journal of Viral Hepatitis, vol. 7, no. 1, pp. 2329, 2000.

[39] D. Sansonno, L. Gesualdo, C. Manno, F. P. Schena, and F. Dammacco, "Hepatitis C virus-related proteins in kidney tissue from hepatitis $\mathrm{C}$ virus-infected patients with cryoglobulinemic membranoproliferative glomerulonephritis," Hepatology, vol. 25, no. 5, pp. 1237-1244, 1997.

[40] C. E. Alpers and J. Kowalewska, "Emerging paradigms in the renal pathology of viral diseases," Clinical Journal of the American Society of Nephrology, vol. 2, supplement 1, pp. S6S12, 2007.

[41] G. D'Amico, "Renal involvement in hepatitis C infection: cryoglobulinemic glomerulonephritis," Kidney International, vol. 54, no. 2, pp. 650-671, 1998.

[42] G. D’Amico and A. Fornasieri, "Cryoglobulinemic glomerulonephritis: a membranoproliferative glomerulonephritis induced by hepatitis C virus," American Journal of Kidney Diseases, vol. 25, no. 3, pp. 361-369, 1995.
[43] M. Wörnle, H. Schmid, B. Banas et al., "Novel role of toll-like receptor 3 in hepatitis C-associated glomerulonephritis," The American Journal of Pathology, vol. 168, no. 2, pp. 370-385, 2006.

[44] A. A. Abou-Zeid and H. K. El-Sayegh, “Toll-like receptor 3 gene expression in Egyptian patients with glomerulonephritis and hepatitis C virus infection," Scandinavian Journal of Clinical and Laboratory Investigation, vol. 71, no. 6, pp. 456-461, 2011.

[45] T. Kawaguchi, T. Yoshida, M. Harada et al., "Hepatitis C virus down-regulates insulin receptor substrates 1 and 2 through upregulation of suppressor of cytokine signaling 3," American Journal of Pathology, vol. 165, no. 5, pp. 1499-1508, 2004.

[46] Y. Shintani, H. Fujie, H. Miyoshi et al., " Hepatitis C virus infection and diabetes. Direct involvement of the virus in the development of insulin resistance," Gastroenterology, vol. 126, no. 3, pp. 840-848, 2004.

[47] F. Su, C. Su, S. Chang et al., "Association of hepatitis c virus infection with risk of ESRD: a population-based study," The American Journal of Kidney Diseases, vol. 60, no. 4, pp. 553-560, 2012.

[48] J. N. Hofmann, A. Törner, W. Chow, W. Ye, M. P. Purdue, and A. Duberg, "Risk of kidney cancer and chronic kidney disease in relation to hepatitis $C$ virus infection: a nationwide registerbased cohort study in Sweden," European Journal of Cancer Prevention, vol. 20, no. 4, pp. 326-330, 2011.

[49] Y. C. Chen, H. Y. Lin, C. Y. Li, M. S. Lee, and Y. C. Su, "A nationwide cohort study suggests that hepatitis $\mathrm{C}$ virus infection is associated with increased risk of chronic kidney disease," Kidney International, vol. 85, no. 5, pp. 1200-1207, 2014.

[50] J. Lee, M. Lin, Y. Yang, S. Lu, H. Chen, and S. Hwang, "Association of hepatitis $\mathrm{C}$ and $\mathrm{B}$ virus infection with CKD in an endemic area in Taiwan: a cross-sectional study," American Journal of Kidney Diseases, vol. 56, no. 1, pp. 23-31, 2010.

[51] L. S. Dalrymple, T. Koepsell, J. Sampson et al., "Hepatitis C virus infection and the prevalence of renal insufficiency," Clinical Journal of the American Society of Nephrology, vol. 2, no. 4, pp. 715-721, 2007.

[52] M. Lin, Y. Chiu, C. Lee et al., "Factors associated with chronic kidney disease in the elderly and nonelderly population," Clinical Journal of the American Society of Nephrology, vol. 8, pp. 33-40, 2013.

[53] J. Soma, T. Saito, Y. Taguma et al., "High prevalence and adverse effect of hepatitis $\mathrm{C}$ virus infection in type II diabetic-related nephropathy," Journal of the American Society of Nephrology, vol. 11, no. 4, pp. 690-699, 2000.

[54] A. Mocroft, J. Neuhaus, L. Peters et al., "Hepatitis B and C coinfection are independent predictors of progressive kidney disease in hiv-positive, antiretroviral-treated adults," PLOS ONE, vol. 7, no. 7, Article ID e40245, 2012.

[55] Y. Arase, F. Suzuki, Y. Kawamura et al., "Development rate of chronic kidney disease in hepatitis $C$ virus patients with advanced fibrosis after interferon therapy," Hepatology Research, vol. 41, no. 10, pp. 946-954, 2011.

[56] F. Fabrizi, V. Dixit, and P. Messa, "Impact of hepatitis C on survival in dialysis patients: a link with cardiovascular mortality?" Journal of Viral Hepatitis, vol. 19, no. 9, pp. 601-607, 2012.

[57] B. Yelken, N. Gorgulu, Y. Caliskan et al., "Association between chronic hepatitis $\mathrm{C}$ infection and coronary flow reserve in dialysis patients with failed renal allografts," Transplantation Proceedings, vol. 41, no. 5, pp. 1519-1523, 2009. 
[58] N. Oyake, T. Shimada, Y. Murakami et al., "Hepatitis C virus infection as a risk factor for increased aortic stiffness and cardiovascular events in dialysis patients," Journal of Nephrology, vol. 21, no. 3, pp. 345-353, 2008.

[59] S. Petta, F. Macaluso, and A. Craxi, "Cardiovascular diseases and HCV infection: a simple association or more?" Gut, vol. 63, no. 3, pp. 369-375, 2013.

[60] M. Lee, H. Yang, S. Lu et al., "Chronic hepatitis C virus infection increases mortality from hepatic and extrahepatic diseases: a community-based long-term prospective study," The Journal of Infectious Diseases, vol. 206, no. 4, pp. 469-477, 2012.

[61] C. Liao, T. Su, F. Sung, W. Chou, and T. Chen, "Does hepatitis $\mathrm{C}$ virus infection increase risk for stroke? A population-based cohort study," PLoS ONE, vol. 7, no. 2, Article ID e31527, 2012.

[62] M. Lee, H. Yang, C. Wang et al., "Hepatitis C virus infection and increased risk of cerebrovascular disease," Stroke, vol. 41, no. 12, pp. 2894-2900, 2010.

[63] Y. Hsu, J. Lin, H. Ho et al., "Antiviral treatment for hepatitis C virus infection is associated with improved renal and cardiovascular outcomes in diabetic patients," Hepatology, vol. 59, no. 4, pp. 1293-1302, 2014.

[64] A. A. Butt, W. Xiaoqiang, M. Budoff, D. Leaf, L. H. Kuller, and A. C. Justice, "Hepatitis $\mathrm{C}$ virus infection and the risk of coronary disease," Clinical Infectious Diseases, vol. 49, no. 2, pp. 225-232, 2009.

[65] C.-S. Hsu, J.-H. Kao, Y.-C. Chao et al., "Interferon-based therapy reduces risk of stroke in chronic hepatitis $\mathrm{C}$ patients: a population-based cohort study in Taiwan," Alimentary Pharmacology \& Therapeutics, vol. 38, no. 4, pp. 415-423, 2013.

[66] A. M. Guiltinan, Z. Kaidarova, B. Custer et al., "Increased allcause, liver, and cardiac mortality among hepatitis $\mathrm{C}$ virusseropositive blood donors," American Journal of Epidemiology, vol. 167, no. 6, pp. 743-750, 2008.

[67] K. A. Forde, K. Haynes, A. B. Troxel et al., "Risk of myocardial infarction associated with chronic hepatitis $\mathrm{C}$ virus infection: a population-based cohort study, Journal of Viral Hepatitis, vol. 19, no. 4, pp. 271-277, 2012.

[68] Y. Ishizaka, N. Ishizaka, E. Takahashi et al., "Association between hepatitis $\mathrm{C}$ virus core protein and carotid atherosclerosis," Circulation Journal, vol. 67, no. 1, pp. 26-30, 2003.

[69] L. E. Adinolfi, L. Restivo, R. Zampino et al., "Chronic HCV infection is a risk of atherosclerosis. Role of HCV and HCVrelated steatosis," Atherosclerosis, vol. 221, no. 2, pp. 496-502, 2012.

[70] S. Petta, D. Torres, G. Fazio et al., "Carotid atherosclerosis and chronic hepatitis C: a prospective study of risk associations," Hepatology, vol. 55, no. 5, pp. 1317-1323, 2012.

[71] A. Mostafa, M. K. Mohamed, M. Saeed et al., "Hepatitis C infection and clearance: impact on atherosclerosis and cardiometabolic risk factors," Gut, vol. 59, no. 8, pp. 1135-1140, 2010.

[72] C. Vassalle, S. Masini, F. Bianchi, and G. C. Zucchelli, "Evidence for association between hepatitis $\mathrm{C}$ virus seropositivity and coronary artery disease," Heart, vol. 90, no. 5, pp. 565-566, 2004.

[73] O. Alyan, F. Kacmaz, O. Ozdemir et al., "Hepatitis C infection is associated with increased coronary artery atherosclerosis defined by modified reardon severity score system," Circulation Journal, vol. 72, no. 12, pp. 1960-1965, 2008.

[74] Z. M. Younossi, M. Stepanova, F. Nader, and E. Elsheikh, "Associations of chronic hepatitis $\mathrm{C}$ with metabolic and cardiac outcomes," Alimentary Pharmacology and Therapeutics, vol. 37, no. 6, pp. 647-652, 2013.
[75] S. Maruyama, M. Koda, N. Oyake et al., "Myocardial injury in patients with chronic hepatitis C infection," Journal of Hepatology, vol. 58, no. 1, pp. 11-15, 2013.

[76] F. Bilora, E. Campagnolo, R. Rinaldi, A. Rossato, M. Arzenton, and F. Petrobelli, "Carotid and femoral atherosclerosis in chronic hepatitis C: a 5-year follow-up," Angiology, vol. 59, no. 6, pp. 717-720, 2009.

[77] M. Masia, S. Padilla, C. Robledano, J. M. Ramos, and F. Gutierrez, "Evaluation of endothelial function and subclinical atherosclerosis in association with hepatitis $\mathrm{C}$ virus in HIVinfected patients. A cross-sectional study," BMC Infectious Diseases, vol. 11, article 265, 2011.

[78] C. M. Arcari, K. E. Nelson, D. M. Netski, F. J. Nieto, and C. A. Gaydos, "No association between hepatitis C virus seropositivity and acute myocardial infarction," Clinical Infectious Diseases, vol. 43, no. 6, pp. e53-e56, 2006.

[79] H. Völzke, C. Schwahn, B. Wolff et al., "Hepatitis B and C virus infection and the risk of atherosclerosis in a general population," Atherosclerosis, vol. 174, no. 1, pp. 99-103, 2004.

[80] M. Shaheen, D. Echeverry, M. G. Oblad, M. I. Montoya, S. Teklehaimanot, and A. J. Akhtar, "Hepatitis C, metabolic syndrome, and inflammatory markers: results from the Third National Health and Nutrition Examination Survey [NHANES III]," Diabetes Research and Clinical Practice, vol. 75, no. 3, pp. 320-326, 2007.

[81] C. Wang, S. Wang, W. Yao, T. Chang, and P. Chou, "Hepatitis $\mathrm{C}$ virus infection and the development of type 2 diabetes in a community-based longitudinal study," American Journal of Epidemiology, vol. 166, no. 2, pp. 196-203, 2007.

[82] E. Bugianesi, F. Salamone, and F. Negro, "The interaction of metabolic factors with HCV infection: does it matter?" Journal of Hepatology, vol. 56, supplement 1, pp. S56-S65, 2012.

[83] M. Pansuria, H. Xi, L. Li, X. Yang, and H. Wang, "Insulin resistance, metabolic stress, and atherosclerosis," Frontiers in Bioscience, vol. 4, pp. 916-931, 2012.

[84] G. Pasterkamp, "Methods of accelerated atherosclerosis in diabetic patients," Heart, vol. 99, no. 10, pp. 743-749, 2013.

[85] M. Boddi, R. Abbate, B. Chellini et al., "Hepatitis C virus RNA localization in human carotid plaques," Journal of Clinical Virology, vol. 47, no. 1, pp. 72-75, 2010. 

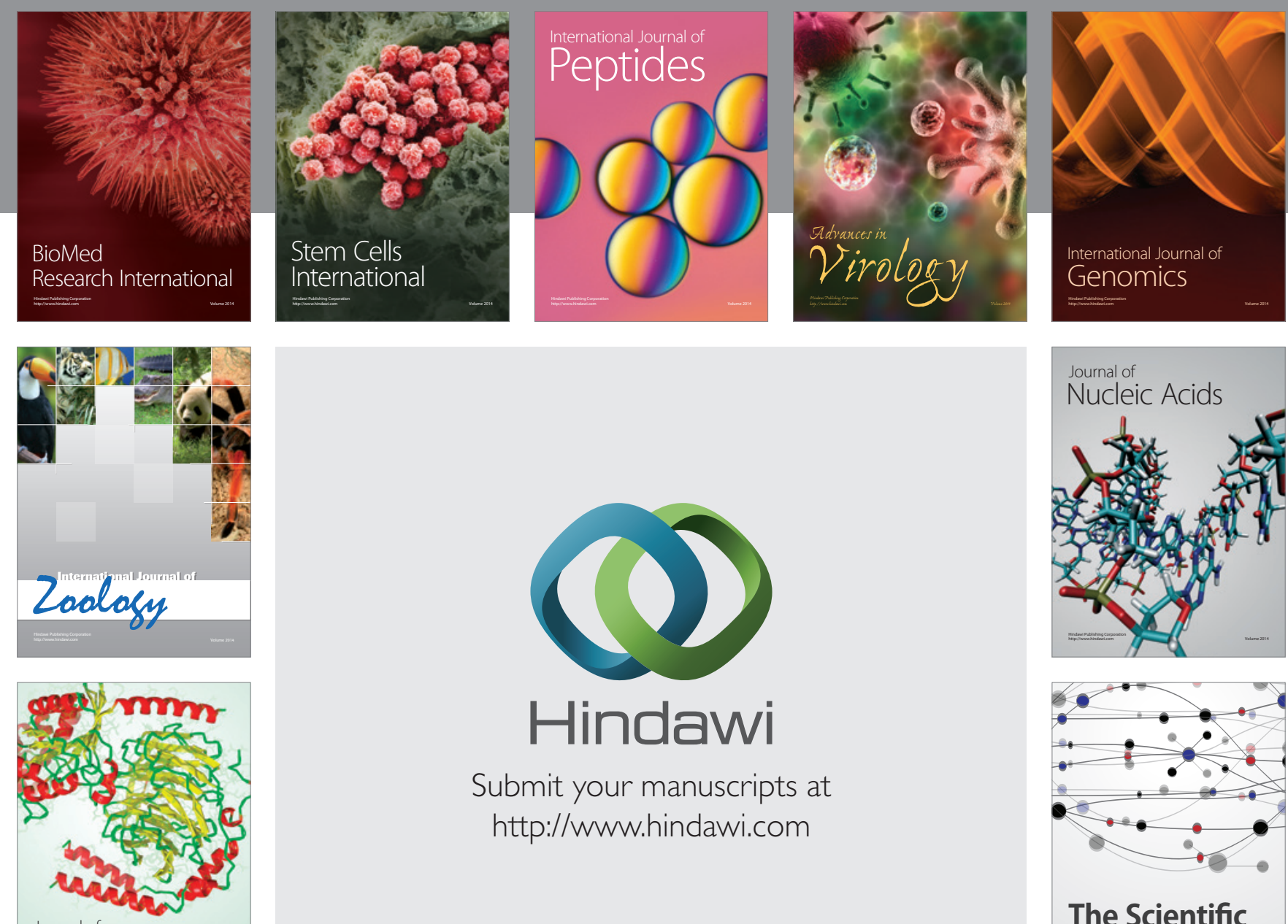

Submit your manuscripts at

http://www.hindawi.com

Journal of
Signal Transduction
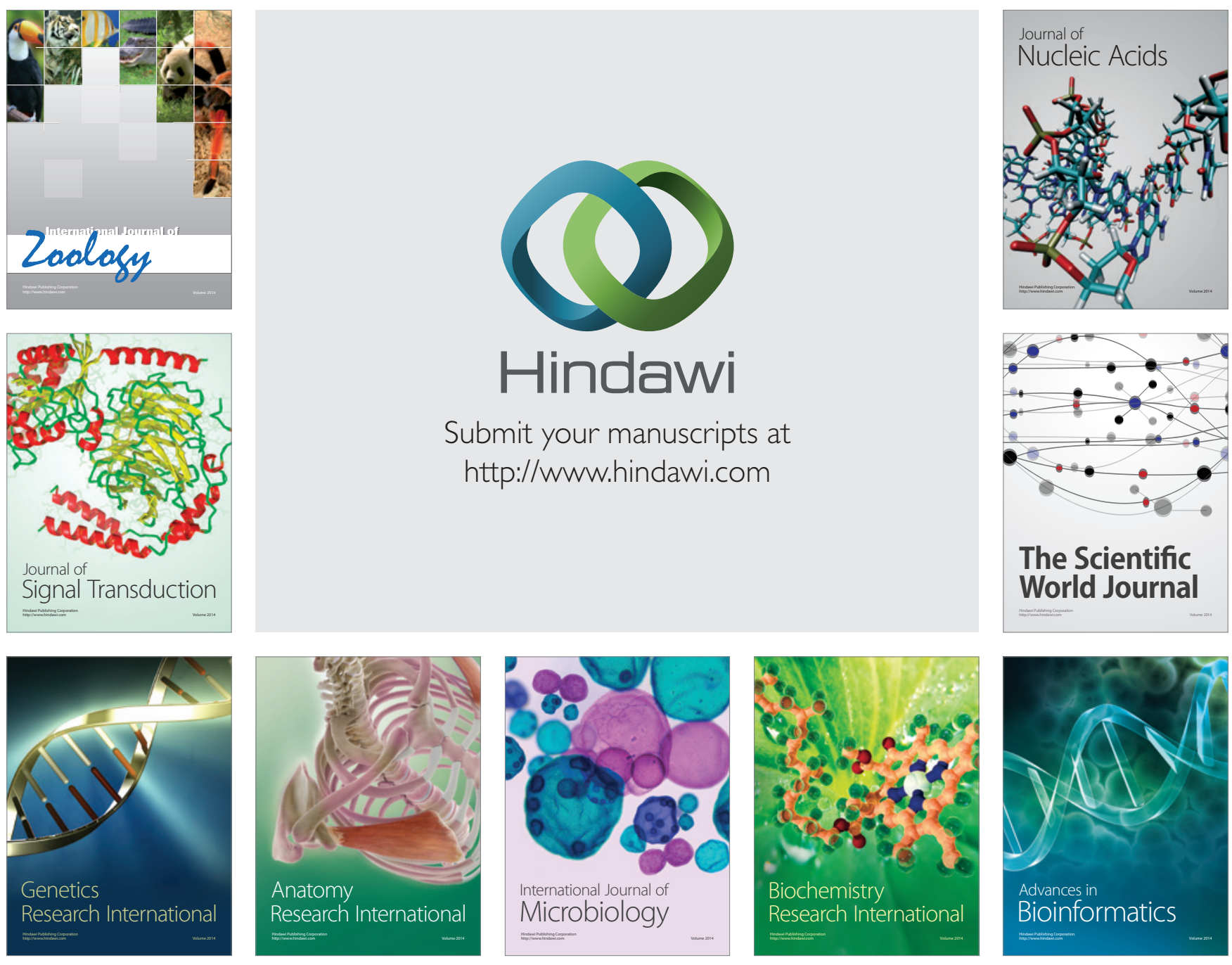

The Scientific World Journal
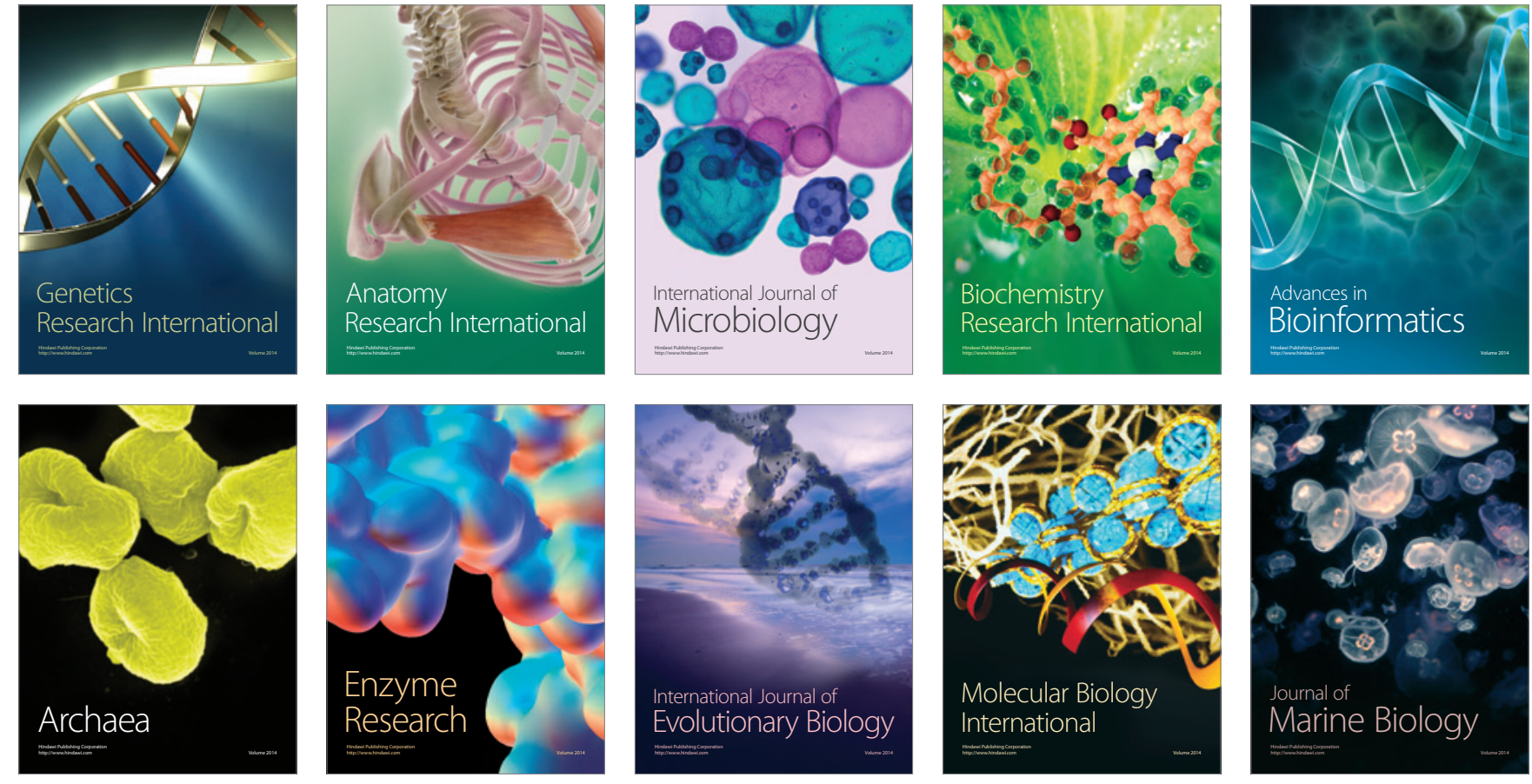